\title{
New Common Fixed Point Results for Four Maps on Cone Metric Spaces"
}

\author{
Yan Han, Shaoyuan Xu \\ School of Mathematics and Statistics, Hubei Normal University, Huangshi, China \\ E-mail: hanyan702@126.com \\ Received June 6, 2011; revised July 2, 2011; accepted July 9, 2011
}

\begin{abstract}
In this paper, some new existence and uniqueness of common fixed points for four mappings are obtained, which do not satisfy continuity and commutation on non-normal cone metric spaces. These results improve and generalize several well-known comparable results in the literature.
\end{abstract}

Keywords: Common Fixed Point, Cone Metric Space, Non-Normal Cone

\section{Introduction and Preliminaries}

Since Huang and Zhang [1] introduced the concept of cone metric space, the study of common fixed points of mappings satisfying certain contractive conditions on cone metric spaces has been at the center of strong research activity, because it has not only important theoretical meaning but also wide applications. Recently, some authors obtained a number of meaningful consulting fixed point theorems for one or two mappings on cone metric spaces(see [1-3,5-8]). The aim of this paper is to present coincidence points results for four mappings without satisfying the notion of continuity and commutation on non-normal cone metric spaces. Common fixed point theorems are obtained under weakly compatible maps. Our results generalized and unified these main results in [1-5].

We recall some definitions and properties of cone metric spaces in [1]. Let $E$ be a real Banach space and $P$ be a subset of $E, \theta$ denotes the zero element of $E$ and int $P$ denotes the interior of $P$. The subset $P$ is called a cone if and only if

1) $P$ is closed, nonempty and $P \neq\{\theta\}$,

2) $a, b \in$ ú, $a, b \geq 0, x, y \in P \Rightarrow a x+b y \in P$,

3) $x \in P$ and $-x \in P \Rightarrow x=\theta$.

Given a cone $P \subset E$, we define a partial ordering $\leq$ with respect to $P$ by $x \leq y$ if and only if $y-x \in P$. We shall write $x<y$ if $x \leq y$ and $x \neq y$, while $x \ll y$ will stand for $y-x \in \operatorname{int} P$. A cone $P$ is called normal if there is a number $N>0$ such that for all $x, y \in P$,

$$
\theta \leq x \leq y \text { implies }\|x\| \leq N\|y\| \text {. }
$$

*This paper is supported by the Foundation of Education Ministry, Hubei Province, China (No: D20102502).
The least positive number satisfying the above inequality is called the normal constant of $P$.

Definition 1.1. ([1]) Let $X$ be a nonempty set. Suppose that the mapping $d: X \times X \rightarrow E$ satisfies:

(d1) $\theta \leq d(x, y)$ for all $x, y \in X$ and $d(x, y)=\theta$ if and only if $x=y$;

(d2) $d(x, y)=d(y, x)$ for all $x, y \in X$;

(d3) $d(x, y) \leq d(x, z)+d(z, y)$ for all $x, y, z \in X$.

Then $d$ is called a cone metric on $X$ and $(X, d)$ is called a cone metric space. It is clear that the cone metric space is more general than metric space.

Definition 1.2 ([1]) Let $(X, d)$ be a cone metric space. Then we say that $\left\{x_{n}\right\}$ is:

1) a Cauchy sequence if for every $c \in E$ with $c \gg \theta$, there is $N$ such that for all $n, m>N, d\left(x_{n}, x_{m}\right) \ll c$;

2) a convergent sequence if for every $c \in E$ with $c \gg \theta$, there is $N$ such that for all $m>N, d\left(x_{m}, x\right) \ll c$ for some fixed $x$ in $X$.

A cone metric space $X$ is said to be complete if every Cauchy sequence in $X$ is convergent in $X$.

Definition 1.3. ([2]) Let $f$ and $g$ be self maps of a set $X$. If $w=f x=g x$ for some $x$ in $X$, then $x$ is called a coincidence point of $f$ and $g$, and $w$ is called a point of coincidence of $f$ and $g$.

Definition 1.4. ([6]) The mappings $f, g: X \rightarrow X$ are weakly compatible if, for every $x \in X$, holds $f g x=g g x$ whenever $f x=g x$.

Lemma 1.5. ([2]) Let $f$ and $g$ be weakly compatible self maps of a set $X$. If $f$ and $g$ have a unique point of coincidence $w=f x=g x$, then $w$ is the unique common fixed point of $f$ and $g$.

Remark 1.6. Let $(X, d)$ be a cone metric space with a cone $P$. If $d(x, y) \leq h d(x, y)$ for all 
$x, y \in X, h \in(0,1)$, then $d(x, y)=\theta$, which implies that $x=y$.

\section{Main Results}

In this section, we give some common fixed point theorems for four mappings defined on a cone metric space. Normality of the cone is not assumed.

Theorem 2.1. Let $(X, d)$ be a cone metric space. Suppose mappings $S, I, T, J: X \rightarrow X$ satisfy

$$
\begin{aligned}
d(S x, T y) & \leq a_{1} d(I x, J y)+a_{2} d(I x, S x) \\
& +a_{3} d(J y, T y)+a_{4} d(I x, T y)+a_{5} d(J y, S x)
\end{aligned}
$$

for all $x, y \in X$, where $a_{i} \geq 0(i=1,2,3,4,5)$ satisfying

$$
\begin{aligned}
& a_{1}+a_{2}+a_{3}+2 \max \left\{a_{4}, a_{5}\right\}<1 \\
& \text { or } a_{1}+2 \max \left\{a_{2}, a_{3}\right\}+a_{4}+a_{5}<1 .
\end{aligned}
$$

If $S(X) \subseteq J(X), T(X) \subseteq I(X)$ and one of $S(X), I(X), J(X)$ and $T(X)$ is a complete subspace of $X$, then the four mappings $S, I, T$ and $J$ have a unique point of coincidence in $X$. Moreover, if $\{T, J\}$ and $\{S, I\}$ are weakly compatible, respectively, all of the mappings $S, I, T$ and $J$ have a unique common fixed point.

Proof. Suppose $x_{0}$ is an arbitrary point in $X$. Since $S(X) \subseteq J(X), T(X) \subseteq I(X)$, there exist $x_{1}, x_{2} \in X$ such that $S x_{0}=J x_{1}, T x_{1}=I x_{2}$. Continuing this process, we can define $\left\{x_{n}\right\}$ by

$$
\begin{gathered}
J x_{2 n+1}=S x_{2 n}, I x_{2 n+2}=T x_{2 n+1}, \quad n=0,1,2, \cdots \text { Denote } \\
y_{2 n}=J x_{2 n+1}=S x_{2 n}, \quad y_{2 n+1}=I x_{2 n+2}=T x_{2 n+1}, \\
n=0,1,2, \cdots
\end{gathered}
$$

Now, we shall show that $\left\{y_{n}\right\}$ is a Cauchy sequence. From (2.1), we know

$$
\begin{aligned}
& d\left(y_{2 n}, y_{2 n+1}\right)=d\left(S x_{2 n}, T x_{2 n+1}\right) \\
& \leq a_{1} d\left(I x_{2 n}, J x_{2 n+1}\right)+a_{2} d\left(I x_{2 n}, S x_{2 n}\right)+a_{3} d\left(J x_{2 n+1}, T x_{2 n+1}\right) \\
& +a_{4} d\left(I x_{2 n}, T x_{2 n+1}\right)+a_{5} d\left(J x_{2 n+1}, S x_{2 n}\right) \\
& \leq a_{1} d\left(y_{2 n-1}, y_{2 n}\right)+a_{2} d\left(y_{2 n-1}, y_{2 n}\right)+a_{3} d\left(y_{2 n}, y_{2 n+1}\right) \\
& +a_{4}\left[d\left(y_{2 n-1}, y_{2 n}\right)+d\left(y_{2 n}, y_{2 n+1}\right)\right]+a_{5} d\left(y_{2 n}, y_{2 n}\right),
\end{aligned}
$$

which implies that

$$
d\left(y_{2 n+1}, y_{2 n}\right) \leq \frac{a_{1}+a_{2}+a_{4}}{1-a_{3}-a_{4}} d\left(y_{2 n}, y_{2 n-1}\right) .
$$

Similarly it is not difficult to show that

$$
\begin{aligned}
& d\left(y_{2 n+2}, y_{2 n+1}\right)=d\left(S x_{2 n+2}, T x_{2 n+1}\right) \\
& \leq a_{1} d\left(I x_{2 n+2}, J x_{2 n+1}\right)+a_{2} d\left(I x_{2 n+2}, S x_{2 n+2}\right) \\
& +a_{3} d\left(J x_{2 n+1}, T x_{2 n+1}\right) \\
& +a_{4} d\left(I x_{2 n+2}, T x_{2 n+1}\right)+a_{5} d\left(J x_{2 n+1}, S x_{2 n+2}\right) \\
& \leq a_{1} d\left(y_{2 n}, y_{2 n+1}\right)+a_{2} d\left(y_{2 n+1}, y_{2 n+2}\right)+a_{3} d\left(y_{2 n}, y_{2 n+1}\right) \\
& +a_{5}\left[d\left(y_{2 n}, y_{2 n+1}\right)+d\left(y_{2 n+1}, y_{2 n+2}\right)\right],
\end{aligned}
$$

which implies that

$$
d\left(y_{2 n+2}, y_{2 n+1}\right) \leq \frac{a_{1}+a_{3}+a_{5}}{1-a_{2}-a_{5}} d\left(y_{2 n+1}, y_{2 n}\right) .
$$

Now, from (2.2) and (2.3), we deduce that

$$
\begin{aligned}
& d\left(y_{2 n+2}, y_{2 n+1}\right) \leq \frac{a_{1}+a_{3}+a_{5}}{1-a_{2}-a_{5}} d\left(y_{2 n+1}, y_{2 n}\right) \\
& \leq \frac{a_{1}+a_{3}+a_{5}}{1-a_{2}-a_{5}} \cdot \frac{a_{1}+a_{2}+a_{4}}{1-a_{3}-a_{4}} d\left(y_{2 n}, y_{2 n-1}\right) \\
& \leq \frac{a_{1}+a_{3}+a_{5}}{1-a_{2}-a_{5}} \cdot \frac{a_{1}+a_{2}+a_{4}}{1-a_{3}-a_{4}} \cdot \frac{a_{1}+a_{3}+a_{5}}{1-a_{2}-a_{5}} d\left(y_{2 n-1}, y_{2 n-2}\right) \\
& \leq \cdots \leq \frac{a_{1}+a_{3}+a_{5}}{1-a_{2}-a_{5}} \cdot\left(\frac{a_{1}+a_{2}+a_{4}}{1-a_{3}-a_{4}} \cdot \frac{a_{1}+a_{3}+a_{5}}{1-a_{2}-a_{5}}\right)^{n} d\left(y_{1}, y_{0}\right)
\end{aligned}
$$

and

$$
\begin{aligned}
& d\left(y_{2 n+3}, y_{2 n+2}\right) \leq \frac{a_{1}+a_{2}+a_{4}}{1-a_{3}-a_{4}} d\left(y_{2 n+2}, y_{2 n+1}\right) \\
& \leq \cdots \leq\left(\frac{a_{1}+a_{2}+a_{4}}{1-a_{3}-a_{4}} \cdot \frac{a_{1}+a_{3}+a_{5}}{1-a_{2}-a_{5}}\right)^{n+1} d\left(y_{1}, y_{0}\right) .
\end{aligned}
$$

Let

$$
M=\frac{a_{1}+a_{2}+a_{4}}{1-a_{3}-a_{4}}, \quad N=\frac{a_{1}+a_{3}+a_{5}}{1-a_{2}-a_{5}} .
$$

If $a_{1}+a_{2}+a_{3}+2 \max \left\{a_{4}, a_{5}\right\}<1$, then

$$
M N=\frac{a_{1}+a_{2}+a_{4}}{1-a_{3}-a_{4}} \cdot \frac{a_{1}+a_{3}+a_{5}}{1-a_{2}-a_{5}}<1 \cdot 1=1 .
$$

If $a_{1}+2 \max \left\{a_{2}, a_{3}\right\}+a_{4}+a_{5}<1$, then

$$
\begin{aligned}
M N & =\frac{a_{1}+a_{2}+a_{4}}{1-a_{3}-a_{4}} \cdot \frac{a_{1}+a_{3}+a_{5}}{1-a_{2}-a_{5}} \\
& =\frac{a_{1}+a_{3}+a_{5}}{1-a_{3}-a_{4}} \cdot \frac{a_{1}+a_{2}+a_{4}}{1-a_{2}-a_{5}}<1 \cdot 1=1 .
\end{aligned}
$$

Now, for any $n>m$, we have

$$
\begin{aligned}
& d\left(y_{2 n+1}, y_{2 m+1}\right) \\
& \leq d\left(y_{2 n+1}, y_{2 n}\right)+d\left(y_{2 n}, y_{2 n-1}\right)+\cdots+d\left(y_{2 m+2}, y_{2 m+1}\right) \\
& \leq\left(\sum_{i=m+1}^{n}(M N)^{i}+N \sum_{i=m}^{n-1}(M N)^{i}\right) d\left(y_{1}, y_{0}\right) \\
& \leq\left(\frac{(M N)^{m+1}}{1-M N}+\frac{N(M N)^{m}}{1-M N}\right) d\left(y_{1}, y_{0}\right) \\
& =(M+1) \frac{N(M N)^{m}}{1-M N} d\left(y_{1}, y_{0}\right) .
\end{aligned}
$$

In analogous way, we gain

$$
d\left(y_{2 n}, y_{2 m+1}\right) \leq(M+1) \frac{N(M N)^{m}}{1-M N} d\left(y_{1}, y_{0}\right),
$$




$$
d\left(y_{2 n}, y_{2 m}\right) \leq(N+1) \frac{(M N)^{m+1}}{1-M N} d\left(y_{1}, y_{0}\right)
$$

and

$$
d\left(y_{2 n+1}, y_{2 m}\right) \leq(N+1) \frac{(M N)^{m+1}}{1-M N} d\left(y_{1}, y_{0}\right) .
$$

Thus, for $n>m>0$

$$
\begin{aligned}
& d\left(y_{n}, y_{m}\right) \\
& \leq \max \left\{(N+1) \frac{(M N)^{m+1}}{1-M N},(M+1) \frac{N(M N)^{m}}{1-M N}\right\} d\left(y_{1}, y_{0}\right) \\
& =\lambda_{m} d\left(y_{1}, y_{0}\right)
\end{aligned}
$$

where $\lambda_{m} \rightarrow 0$ as $m \rightarrow \infty$.

For each $c \gg \theta$, choose $\delta>0$ such that $c-x \in$ int $P$, where $\|x\| \ll \delta$, i.e., $x \ll c$. For this $\delta$, we can choose a natural number $N_{1}$ such that $\left\|\lambda_{m} d\left(y_{1}, y_{0}\right)\right\|<\delta$ for $m>N_{0}$. Thus, we get

$$
d\left(y_{n}, y_{m}\right) \leq \lambda_{m} d\left(y_{1}, y_{0}\right) \ll c \text {, for all } n>m>N_{0} .
$$

Therefore $\left\{y_{n}\right\}$ is a Cauchy sequence in $(X, d)$. Suppose $J(X)$ is complete, there exists $q \in J(X)$ such that $y_{2 n}=S x_{2 n}=J x_{2 n+1} \rightarrow q$ as $n \rightarrow \infty$. So we can find a $p \in X$ such that $J p=q$. (If $S(X)$ is complete, there exists $q \in S(X) \subseteq J(X)$, then the conclusions remain the same.) Letting $n \rightarrow \infty$, and by $S x_{2 n}=J x_{2 n+1} \rightarrow q$, as $n \rightarrow \infty$, we can choose a natural number $N_{1}$ such that $d\left(y_{2 n}, q\right) \ll \frac{\left(1-a_{3}-a_{4}\right) c}{4}$ and $d\left(y_{2 n}, y_{2 n-1}\right) \ll \frac{\left(1-a_{3}-a_{4}\right) c}{2}$ for $n \geq N_{1}$. Now we show show that $T p=q$. By (2.1), we have

$$
\begin{aligned}
d & (T p, q) \leq d\left(S x_{2 n}, T p\right)+d\left(S x_{2 n}, q\right) \\
\leq & a_{1} d\left(I x_{2 n}, J p\right)+a_{2} d\left(I x_{2 n}, S x_{2 n}\right)+a_{3} d(J p, T p) \\
& +a_{4} d\left(I x_{2 n}, T p\right)+a_{5} d\left(J p, S x_{2 n}\right)+d\left(S x_{2 n}, q\right) \\
= & a_{1} d\left(y_{2 n-1}, q\right)+a_{2} d\left(y_{2 n-1}, y_{2 n}\right)+a_{3} d(q, T p) \\
& +a_{4} d\left(y_{2 n-1}, T p\right)+a_{5} d\left(q, y_{2 n}\right)+d\left(y_{2 n}, q\right) \\
\leq & a_{1}\left[d\left(y_{2 n-1}, y_{2 n}\right)+d\left(y_{2 n}, q\right)\right] \\
& +a_{2} d\left(y_{2 n-1}, y_{2 n}\right)+a_{3} d(q, T p) \\
& +a_{4}\left[d\left(y_{2 n-1}, y_{2 n}\right)+d\left(y_{2 n}, q\right)+d(q, T p)\right] \\
& +\left(a_{5}+1\right) d\left(q, y_{2 n}\right) .
\end{aligned}
$$

Taking $n \rightarrow \infty$, we get

$$
\begin{aligned}
\left(1-a_{3}-a_{4}\right) d(q, T p) \leq & \left(a_{1}+a_{2}+a_{4}\right) d\left(y_{2 n-1}, y_{2 n}\right) \\
& +\left(a_{1}+a_{4}+a_{5}+1\right) d\left(y_{2 n}, q\right) .
\end{aligned}
$$

Then, we get $d(q, T p) \ll \frac{c}{2}+\frac{c}{2}=c$, i.e. $T p=q=J p$.
At the same time, as $q=T p \in T(X) \subseteq I(X)$, there exists $u$ in $X$ such that $I u=q$. From (2.1),

$$
\begin{aligned}
& d(S u, q)=d(S u, T p) \\
\leq & a_{1} d(I u, J p)+a_{2} d(I u, S u)+a_{3} d(J p, T p) \\
& +a_{4} d(I u, T p)+a_{5} d(J p, S u) \\
= & \left(a_{2}+a_{5}\right) d(S u, q) .
\end{aligned}
$$

Hence, from Remark 1.6, we know $S u=q$. Therefore, $T p=J p=q, S u=I u=q$.

Next if we assume $I(X)$ is complete, there exists $q \in I(X)$ such that

$y_{2 n+1}=I x_{2 n+2}=T x_{2 n+1} \rightarrow q$ as $n \rightarrow \infty$. So we can find a $u \in X$ such that $I u=q$. (If $T(X)$ is complete, there exists $q \in T(X) \subseteq I(X)$, then the conclusions remain the same.) Then, we can choose a natural number $N_{2}$

such that $d\left(y_{2 n+1}, q\right) \ll \frac{\left(1-a_{2}-a_{5}\right) c}{4}$ and

$d\left(y_{2 n+1}, y_{2 n}\right) \ll \frac{\left(1-a_{2}-a_{5}\right) c}{2}$ for $n \geq N_{2}$. Now we

show that $S u=q$. By (2.1), we get

$$
\begin{aligned}
d & (S u, q) \leq d\left(S u, T x_{2 n+1}\right)+d\left(T x_{2 n+1}, q\right) \\
\leq & a_{1} d\left(I u, J x_{2 n+1}\right)+a_{2} d(I u, S u) \\
& +a_{3} d\left(J x_{2 n+1}, T x_{2 n+1}\right)+a_{4} d\left(I u, T x_{2 n+1}\right) \\
& +a_{5} d\left(J x_{2 n+1}, S u\right)+d\left(T x_{2 n+1}, q\right) \\
= & a_{1} d\left(y_{2 n}, q\right)+a_{2} d(q, S u)+a_{3} d\left(y_{2 n}, y_{2 n+1}\right) \\
& +a_{4} d\left(q, y_{2 n+1}\right)+a_{5} d\left(y_{2 n}, S u\right)+d\left(y_{2 n+1}, q\right) \\
\leq & a_{1}\left[d\left(y_{2 n+1}, q\right)+d\left(y_{2 n+1}, y_{2 n}\right)\right] \\
& +a_{2} d(q, S u)+a_{3} d\left(y_{2 n+1}, y_{2 n}\right) \\
& +\left(a_{4}+1\right) d\left(q, y_{2 n+1}\right) \\
& +a_{5}\left[d\left(y_{2 n}, y_{2 n+1}\right)+d\left(y_{2 n+1}, q\right)+d(q, S u)\right] .
\end{aligned}
$$

Letting $n \rightarrow \infty$, by Lemma 1.6 we get

$$
\begin{aligned}
& \left(1-a_{2}-a_{5}\right) d(S u, q) \leq\left(a_{1}+a_{3}+a_{5}\right) d\left(y_{2 n+1}, y_{2 n}\right) \\
& \quad+\left(a_{1}+a_{4}+a_{5}+1\right) d\left(y_{2 n+1}, q\right) .
\end{aligned}
$$

Thus, we have $d(q, S u) \ll \frac{c}{2}+\frac{c}{2}=c$, i.e.

$S u=q \in S(X) \subseteq J(X)$, then there exists $p \in X$ such that $J p=q$. In the same method we can also obtain $T p=q$.

Finally, we show that $T$ and $J, S$ and $I$ have a unique point of coincidence in $X$. Assume there exists another point $z \in X$ such that $T x=J x=z$, then

$$
\begin{aligned}
& d(q, z)=d(S u, T x) \leq a_{1} d(I u, J x)+a_{2} d(I u, S u) \\
& +a_{3} d(J x, T x)+a_{4} d(I u, T x)+a_{5} d(J x, S u),
\end{aligned}
$$


which gives

$$
d(q, z) \leq\left(a_{1}+a_{4}+a_{5}\right) d(q, z),
$$

thus $d(q, z)=\theta$, i.e., $T x=T p=J x=J p=q=z$. That is to say, $q$ is a unique point of coincidence in $X$ of $T$ and $J$. Similarly, we also have $q$ is a unique point of coincidence of $S$ and $I$ by induction. So, according to Lemma 1.5, $q$ is the unique common fixed point of $\{T, J\}$ and $\{S, I\}$. Therefore, $q$ is the unique com- mon fixed point of the four mappings $T, J, S$ and $I$. The proof of the theorem is completed.

As a variant of Theorem 2.1, we get the following Corollary.

Corollary 2.2. Let $(X, d)$ be a cone metric space. Suppose mappings $S, I, T, J: X \rightarrow X$ satisfy

$$
\begin{aligned}
& d\left(S^{m} x, T^{n} y\right) \leq a_{1} d\left(I^{m} x, J^{n} y\right)+a_{2} d\left(I^{m} x, S^{m} x\right) \\
& +a_{3} d\left(J^{n} y, T^{n} y\right)+a_{4} d\left(I^{m} x, T^{n} y\right)+a_{5} d\left(J^{n} y, S^{m} x\right)
\end{aligned}
$$

for all $x, y \in X$, where $a_{i} \geq 0(i=1,2,3,4,5)$ satisfying

$$
\begin{aligned}
& a_{1}+a_{2}+a_{3}+2 \max \left\{a_{4}, a_{5}\right\}<1 \\
& \text { or } a_{1}+2 \max \left\{a_{2}, a_{3}\right\}+a_{4}+a_{5}<1 .
\end{aligned}
$$

If $S(X) \subseteq J(X), T(X) \subseteq I(X)$ and one of $S(X), I(X), J(X)$ and $T(X)$ is a complete subspace of $X$, then the four mappings $S, I, T$ and $J$ have a unique point of coincidence in $X$. Moreover, if $\{T, J\}$ and $\{S, I\}$ are weakly compatible, respectively, all of the mappings $S, I, T$ and $J$ have a unique common fixed point.

Proof. It follows from Theorem 2.1, that the four mappings $S^{m}, I^{m}, T^{n}$ and $J^{n}$ have a unique common fixed point $q$. Now, $S q=S\left(S^{m} q\right)=S^{m+1} q=S^{m}(S q)$ and $I q=I\left(I^{m} q\right)=I^{m+1} q=I^{m}(I q)$ implies that $S q$ and $I q$ are also fixed points for $S^{m}$ and $I^{m}$. Hence, $S q=I q=q$. By using the same method, we have $T q=J q=q$.

Remark 2.3. Compared with corresponding results in the literature [4, Theorem 2.8 and Corollary 2.9], Theorem 2.1 show that our hypotheses are greatly weaker than those conditions. On the one hand, we ensure the existence and uniqueness of a common fixed point of four mappings without continuity and commutation; on the other hand, they are special cases of Theorem 2.1 and Corollary 2.2 with $a_{4}=a_{5}$. Hence, Theorem 2.1 present a more general format of common fixed point for four mappings without continuity and commutation on nonnormal cone metric spaces, which extends the main results in [4].

Now we use Theorem 2.1 to obtain a series of new common fixed point theorems for four mappings in non-normal cones metric spaces, which improve and generalize several known results in [1-3].

Corollary 2.4. Let $(X, d)$ be a cone metric space. Suppose mappings $S, I, T, J: X \rightarrow X$ satisfy

$$
\begin{aligned}
& d(S x, T y) \leq \alpha d(I x, J y)+\beta[d(I x, S x)+d(J y, T y)] \\
& +\gamma[d(I x, T y)+d(J y, S x)],
\end{aligned}
$$

for all $x, y \in X$, where $\alpha, \beta, \gamma \geq 0$ and $\alpha+2 \beta+2 \gamma<1$. If $S(X) \subseteq J(X), T(X) \subseteq I(X)$ and one of $S(X), I(X), J(X)$ and $T(X)$ is a complete subspace of $X$, then the four mappings $S, I, T$ and $J$ have a unique point of coincidence in $X$. Moreover, if $\{T, J\}$ and $\{S, I\}$ are weakly compatible, respectively, all of the mappings $S, I, T$ and $J$ have a unique common fixed point.

Proof. Let $a_{1}=\alpha, a_{2}=a_{3}=\beta, a_{4}=a_{5}=\gamma$ in Theorem 2.1.

Corollary 2.5. Let $(X, d)$ be a cone metric space. Suppose mappings $S, I, T, J: X \rightarrow X$ satisfy

$$
d(S x, T y) \leq \alpha d(I x, J y)+\beta[d(I x, S x)+d(J y, T y)],
$$

for all $x, y \in X$, where $\alpha, \beta \geq 0$ and $\alpha+2 \beta<1$. If $S(X) \subseteq J(X), T(X) \subseteq I(X)$ and one of $S(X), I(X), J(X)$ and $T(X)$ is a complete subspace of $X$, then the four mappings $S, I, T$ and $J$ have a unique point of coincidence in $X$. Moreover, if $\{T, J\}$ and $\{S, I\}$ are weakly compatible, respectively, all of the mappings $S, I, T$ and $J$ have a unique common fixed point.

Corollary 2.6. Let $(X, d)$ be a cone metric space. Suppose mappings $S, I, T, J: X \rightarrow X$ satisfy

$$
d(S x, T y) \leq k d(I x, S x)+l d(J y, T y),
$$

for all $x, y \in X$, where $k, l \in[0,1)$ and $k+l<1$. If $S(X) \subseteq J(X), T(X) \subseteq I(X)$ and one of $S(X), I(X), J(X)$ and $T(X)$ is a complete subspace of $X$, then the four mappings $S, I, T$ and $J$ have a unique point of coincidence in $X$. Moreover, if $\{T, J\}$ and $\{S, I\}$ are weakly compatible, respectively, all of the mappings $S, I, T$ and $J$ have a unique common fixed point.

Proof. Let $a_{1}=a_{4}=a_{5}=0, a_{2}=k, a_{3}=l$ in Theorem 2.1, the conclusions are true.

Corollary 2.7. Let $(X, d)$ be a cone metric space. Suppose mappings $S, I, T, J: X \rightarrow X$ satisfy

$$
d(S x, T y) \leq k d(I x, T y)+l d(J y, S x),
$$

for all $x, y \in X$, where $k, l \in[0,1)$ and $k+l<1$. If $S(X) \subseteq J(X), T(X) \subseteq I(X)$ and one of

$S(X), I(X), J(X)$ and $T(X)$ is a complete subspace of $X$, then the four mappings $S, I, T$ and $J$ have a unique point of coincidence in $X$. Moreover, if $\{T, J\}$ and $\{S, I\}$ are weakly compatible, respectively, 
all of the mappings $S, I, T$ and $J$ have a unique common fixed point.

Corollary 2.8. ([4]) Let $(X, d)$ be a cone metric space. Suppose mappings $S, I, T, J: X \rightarrow X$ satisfy

$$
d(S x, T y) \leq k d(I x, J y)
$$

for all $x, y \in X$, where $k \in[0,1)$. If

$S(X) \subseteq J(X), T(X) \subseteq I(X)$ and one of

$S(X), I(X), J(X)$ and $T(X)$ is a complete subspace of $X$, then $\{T, J\}$ and $\{S, I\}$ have a unique point of coincidence in $X$. Moreover, if $\{T, J\}$ and $\{S, I\}$ are weakly compatible, respectively, all of the mappings $S, I, T$ and $J$ have a unique common fixed point.

Remark 2.9. Compared to Theorem 2.1 and Corollary 2.2-2.8 in [3], our Corollary 2.2, 2.4-2.8 do not require that condition " $P$ is a normal cone". Moreover, when we further restrict $I=J=I_{x}$ in Corollary 2.4, which $I_{x}$ is the identity map on $X$, we get Theorem 2.1 in [3], and we get Corollary 2.2-2.8 in [3] when we even define $S=T$ in Corollary 2.2, 2.5-2.8. Hence, Corollary 2.2, 2.4-2.8 improve and generalize Theorem 2.1 and Corollary 2.2-2.8 in [3].

Corollary 2.10. Let $(X, d)$ be a cone metric space. Suppose mappings $f, g: X \rightarrow X$ satisfy

$$
\begin{aligned}
d(f x, f y) \leq & \alpha d(g x, g y)+\beta[d(g x, f x)+d(g y, f y)] \\
& +\gamma[d(g x, f y)+d(g y, f x)]
\end{aligned}
$$

for all $x, y \in X$, where $\alpha, \beta, \gamma \geq 0$ and $\alpha+2 \beta+2 \gamma<1$. If the range of $g$ contains the range of $f$ and $g(X)$ or $f(X)$ is a complete subspace of $X$, then $f$ and $g$ have a unique point of coincidence in $X$. Moreover, if $f$ and $g$ are weakly compatible, $f$ and $g$ have a unique common fixed point.

Proof. Let $S=T=f$ and $I=J=g$ in Corollary 2.4.

Corollary 2.11. Let $(X, d)$ be a cone metric space. Suppose mappings $f, g: X \rightarrow X$ satisfy

$$
\begin{aligned}
& d(f x, f y) \leq a_{1} d(g x, g y)+a_{2} d(f x, g x) \\
& +a_{3} d(f y, g y)+a_{4} d(g x, f y)+a_{5} d(g y, f x),
\end{aligned}
$$

for all $x, y \in X$, where $a_{i} \geq 0(i=1,2,3,4,5)$ and $\sum_{i=1}^{5} a_{i}<1$. If $f(X) \subseteq g(X)$ and $g(X)$ or $f(X)$ is a complete subspace of $X$, then $f$ and $g$ have a unique point of coincidence in $X$. Moreover, if $f$ and $g$ are weakly compatible, $f$ and $g$ have a unique common fixed point.

Proof. In (2.10) interchanging the roles of $x$ and $y$, and adding the new inequality to (2.10), yield (2.9) with $\alpha=a_{1}, \beta=\frac{a_{2}+a_{3}}{2}, \gamma=\frac{a_{4}+a_{5}}{2}$.

Remark 2.12. We note that in our Corollary 2.10 and
Corollary $2.11 g(X)$ or $f(X)$ is complete, and the results remain the same, while Theorem 2.1 in [5] requires that $g(X)$ is complete. In addition, Theorem 2.1 in [5] and Theorem 2.1 in [3] generalize the corresponding results in [1-2]. Therefore, above all, our results improve and unify all of these main results in [1-5].

Remark 2.13. In Theorem 2.1 we do not require mappings $S, T, I$ and $J$ to be compact or continuous. Moreover, we delete the condition " $P$ is a normal cone" in this papers. In addition, when we choose $E=R, P=[0, \infty)$ in the above theorems and corollaries, similar conclusions will be gained in simple metric spaces.

\section{Acknowledgements}

The authors thank the referee for his/her careful reading and useful suggestions of the manuscript.

\section{References}

[1] L. G. Huang and X. Zhang, "Cone Metric Space and Fixed Point Theorems of Contractive Mappings," Journal of Mathematical Analysis and Applications, Vol. 332, No. 2, 2007, pp. 1468-1476. doi:10.1016/j.jmaa.2005.03.087

[2] M. Abbas and G. Jungck, "Common Fixed Point Results for Noncommuting Mappings without Continuity in Cone Metric Spaces," Journal of Mathematical Analysis and Applications, Vol. 341, No. 1, 2008, pp. 416-420. doi:10.1016/j.jmaa.2007.09.070

[3] M. Abbas and B. E. Rhoades, "Fixed and Periodic Point Results in Cone Metric Spaces," Applied Mathematics Letters, Vol. 22, No. 4, 2009, pp. 511-515. doi:10.1016/j.aml.2008.07.001

[4] M. Abbas, B. E. Rhoades and T. Nazir, "Common Fixed Points for Four Maps in Cone Metric Spaces," Applied Mathematics and Computation, Vol. 216, No. 1, 2010, pp. 80-86. doi:10.1016/j.amc.2010.01.003

[5] G. X. Song, X. Y. Sun, Y. Zhao and G. T. Wang, "New Common Fixed Point Theorems for Maps on Cone Metric Spaces," Applied Mathematics Letters, Vol. 23, No. 9, 2010, pp. 1033-1037. doi:10.1016/j.aml.2010.04.032

[6] C. D. Bari and P. Vetro, " $\varphi$-Pairs and Common Fixed Points in Cone Metric Spaces," Rendiconti del Circolo Matematico Palermo, Vol. 57, 2008, pp. 279-285.

[7] S. Radenovic, "Common Fixed Points under Contractive Conditions in Cone Metric Spaces," Computers and Mathematics with Applications, Vol. 58, No. 6, 2009, pp. 1273-1278. doi:10.1016/j.camwa.2009.07.035

[8] Sh. Rezapour and R. Hamlbarani, "Some Note on the Paper "Cone Metric Spaces and Fixed Point Theorems of Contractive Mappings," Journal of Mathematical Analysis and Applications, Vol. 345, No. 2, 2008, pp. 719-724. doi:10.1016/j.jmaa.2008.04.049 